\title{
THE ASSOCIATION OF ERAP2 GENE POLYMORPHISMS WITH SERONEGATIVE SPONDYLOARTHROPATHIES IN HLA-B27 NEGATIVE ROMANIANS
}

\author{
Laura Ioana Cherciu' ${ }^{1}$, Marius Cherciu ${ }^{1}$, Luis Ovidiu Popa ${ }^{2}$, Mihai Bojinca ${ }^{3}$, Monica Irina Dutescu ${ }^{4}$, \\ Violeta Bojinca ${ }^{5}$, Constantin Bara ${ }^{1}$, Olivia Mihaela Popa ${ }^{1}$ \\ ${ }^{\prime}$ Department of Immunology and Pathophysiology, \\ Carol Davila University of Medicine and Pharmacy, Bucharest, Romania \\ ${ }^{2}$ Molecular Biology Department, Grigore Antipa National Museum of Natural History, Bucharest, Romania \\ ${ }^{3}$ Department of Rheumatology, Carol Davila University of Medicine and Pharmacy, \\ I.C. Cantacuzino Hospital, Bucharest, Romania \\ ${ }^{4}$ Prof. Dr. C. T. Nicolau National Institute of Blood Transfusion, Bucharest, Romania \\ ${ }^{5}$ Department of Rheumatology, Carol Davila University of Medicine and Pharmacy, \\ Sf. Maria Hospital, Bucharest, Romania
}

\begin{tabular}{|c|c|}
\hline & Abstract \\
\hline \multicolumn{2}{|r|}{ 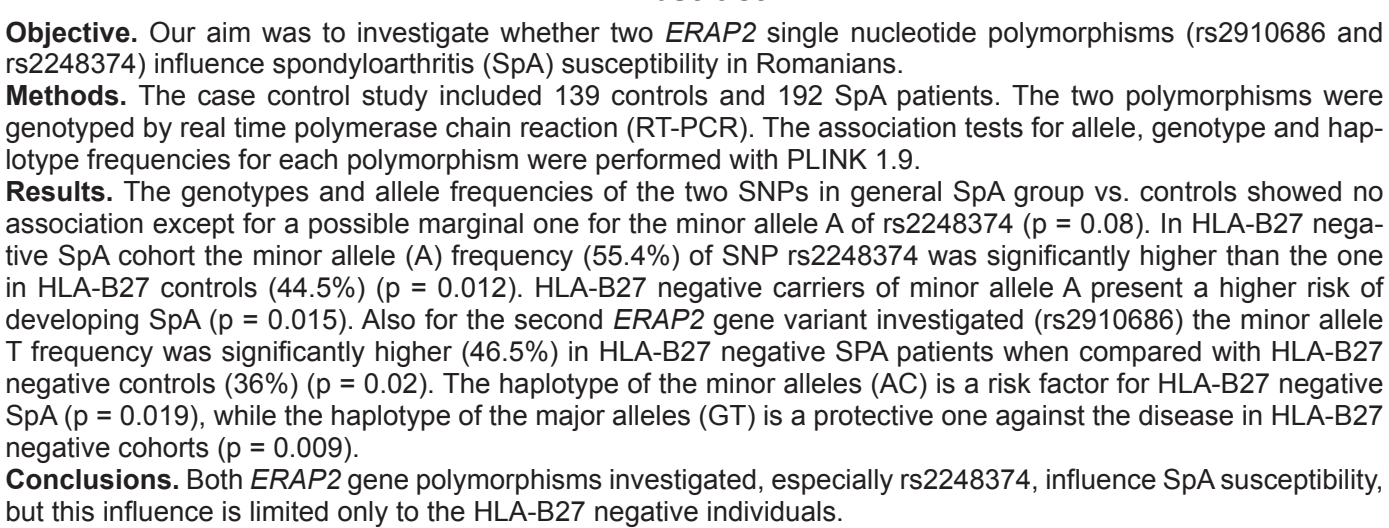 } \\
\hline & \\
\hline
\end{tabular}

\section{INTRODUCTION}

Seronegative spondyloarthropathies or spondyloarthritides are related inflammatory rheumatic diseases with a complex etiopathogeny which in recent years has started to be elucidated, as increasingly more and more genetic association studies have identified new possible candidate genes responsible for influencing disease susceptibility. SpA comprise a central core consisting of ankylosing spondylitis (AS) and psoriatic arthritis (PsA), to which reactive arthritis, arthritis associated with inflammatory bow- el disease and undifferentiated spondyloarthritis (USpA) are added (1). Juvenile SpA sometimes joins this group, because it is considered that $\mathrm{SpA}$ is only an extension of a childhood-onset disease into the adult life (2). Acute anterior uveitis is also classified by some authors among $\mathrm{SpA}$, mainly because about $40 \%$ of patients with uveitis present in fact undiagnosed spondyloarthritis $(3,4)$.

The most emblematic genetic attribute of $\mathrm{SpA}$ is the association with HLA-B27 $(5,6)$. On average $80 \%$ of the axial SpA are HLA-B27 positive $(90 \%$

Correspondence address:

Olivia Mihaela Popa, PhD, Department of Pathophysiology and Immunology, Carol Davila University of Medicine and Pharmacy,

37 Dionisie Lupu Street, Bucharest, Romania

E-mail: oliviapopa@yahoo.com 
for SA (7), 70\% for axial PsA). The percentage decreases to $25 \%$ in peripheral forms of PsA $(8,9)$. Moreover, the variable distribution of HLA-B27 in different populations influences directly the prevalence and incidence of SpA in those populations (7). In Europe one of the highest recorded prevalence $(1.9 \%)$ was in Germany (10), but high values were encountered also in the Azores (1.6\%) (11) and Italy $(1.06 \%)(12)$. Lower values (prevalence of around $0.3 \%$ ) were reported in France (13) and Sweden $(0.45 \%)(14)$. In the United States the estimated prevalence ranges between $0.7 \%$ and $1 \%(15,16)$. With the exception of China, where the prevalence values approach the ones reported in Europe (1719), the SpA prevalence in Asian countries is generally lower (e.g. Japan $=0.0095 \%$ ) (20).

More and more recent genetic association studies have begun to identify non-major histocompatibility complex (MHC) genes that influence the risk of SpA. Single nucleotide polymorphisms (SNPs) belonging to ERAPI and $I L-23 R$ genes were reported to be associated with AS and SpA by several genome wide association studies (GWAS) (21-23). It is currently considered that besides HLA-B27 the ERAP1 gene contributes the most to the AS development, with an attributable risk of $26 \%$ (24). But ERAPI gene's association with AS and $\mathrm{SpA}$ is strictly restricted to HLA-B27 positive individuals $(23,25)$, suggesting the essential cooperation between the molecules encoded by these genes (ERAP1 - the enzyme responsible for processing antigenic peptides and HLA-B27 - the antigen presenting molecule) in AS etiopathogeny.

Although less studied, it seems that ERAP2 gene, which encodes a different aminopeptidase having a similar but at the same time complementary function as ERAP1 in the endoplasmic reticulum, is associated with AS. In 2010 Tsui et al. identified a haplotype combination of three polymorphisms (rs27044 [G] rs30187 [T] rs2549782 [T]), the first two belonging to ERAP1 gene and the last one to ERAP2 gene, as being associated with familial AS (26). A very recent study reported the association of ERAP2 gene with ankylosing spondylitis in HLA-B27 positive and negative patients (27).

ERAP2 belongs to the M1 metallopeptidase family which exerts its proteolytic function via two amino acid motifs (H-E-x-x-H (x) 18-E and G-A-ME-N) in cooperation with the Zinc atom found in their structure (28). ERAP2 has 960 amino acids which are organized in a tertiary structure having many similarities to that of ERAP1 (29). The ERAP2 molecule is encoded by the ERAP2 gene located on the long arm of chromosome 5 in position 15 , in close proximity to ERAPl gene from which it is considered to be recently duplicated (30).

ERAP2 molecule, similarly to ERAP1 molecule, is involved in antigenic processing in the endoplasmic reticulum, from where the processed peptides are transferred onto the MHC I molecules (e.g. HLA-B27) and then exposed on the surface of antigen-presenting cells. Therefore, the aminopeptidase activity of both molecules directly affect the antigenic profile, which activate the CD8+ T lymphocytes and natural killer cells (31-33). Saveanu et al. have identified the existence of an ERAP1/ERAP2 heterodimer which provides partial proteolysis highly effective to the peptide sequences derived from proteosome (34).

Although ERAP1 can process efficiently long chain peptides (12 to 16 amino acids), which are shortened to the optimum length of 8-9 amino acids, it may have difficulties when it comes to peptides smaller than eight amino acids not capable of interacting with the regulatory site, and therefore it cannot activate the catalytic site of ERAP1 (35). It seems that ERAP2 compensates for this limitation and it can be activated by smaller peptides of 7-8 amino acids (36). Recently, Mpakali et al. have hypothesized that ERAP2 operates as an accessory aminopeptidase for ERAP1, processing the peptides inaccessible to ERAP1, but also proteolyzing completely certain potential epitopes (36).

The scientific literature regarding the ERAP2 gene association with AS is not as extended as the one regarding ERAP1 association with AS. Moreover, to date, there are no studies (indexed in PubMed database) investigating the association of ERAP2 gene polymorphisms with SpA.

The objective of this study was to investigate two ERAP2 single nucleotide polymorphisms (rs2910686 and rs2248374) in relation to disease susceptibility in Romanian patients with $\mathrm{SpA}$.

\section{MATERIALS AND METHODS}

\section{Patients and controls}

The case-control study included 139 healthy controls and 192 patients with seronegative spondyloarthritis (80 AS patients, 99 PsA patients and 13 USpA patients). 
The consecutively recruited patients were diagnosed in the Departments of Rheumatology and Internal Medicine of Dr. I. Cantacuzino Hospital and Sf. Maria Hospital in Bucharest. The selection criteria for those patients enrolled after 2009/2011 were based on ASAS classification criteria for axial and peripheral SpA (37-39), while the ESSG criteria were used for the previously recruited patients (40). The 80 AS patients fulfilled also the 1984 modified New York criteria (41), and for PsA patients the inclusion criteria relied on the most recent criteria for psoriatic arthritis (CASPAR, 2006) (42). The diagnosis of undifferentiated spondyloarthritis was sustained when the patients fulfilled the ASAS/ESSG criteria for SpA without meeting the 1984 modified New York criteria, CASPAR criteria or the criteria for inflammatory bowel disease and reactive arthritis. The cohort of healthy controls included subjects of Romanian ethnicity without symptoms or signs of arthritis and without a history of rheumatic disease. Subjects were selected from potential bone marrow donors registered by the Prof. Dr. C.T. Nicolau National Institute of Blood Transfusion, Bucharest. In the control group the median age was 35.5 years and the ratio of male: female was 71:68, while in the SpA cohort the median age was 46.8 years and male: female ratio was 116:76 (Table 1).

TABLE 1. Cohorts included in the study: main characteristics

\begin{tabular}{|l|c|c|}
\hline Characteristics & SpA patients & Healthy controls \\
\hline Male/female ratio & $116: 76$ & $71: 68$ \\
\hline Median age & 46.8 & 35.5 \\
\hline Mean age & 47 & 37.8 \\
\hline $\begin{array}{l}\text { HLA-B27 } \\
\text { positive : negative ratio }\end{array}$ & $91: 101$ & $13: 118$ \\
\hline
\end{tabular}

HLA-B27+, human leukocyte antigen B27 positive; SpA, spondyloarthritides.

Patients and controls included in the study agreed to participate and signed an informed consent for genetic testing, which explained the quasi-absence of the risk involved (the risk of phlebotomy itself) and the potential future benefits related to the more precise genetic characterization of $\mathrm{SpA}$, the study receiving the approval of the local ethics committees.

The genomic DNA extraction was performed using commercial genomic DNA kits: Blood me Isolation Kit, Metabion, Germany and QIAamp DNA Blood Mini Kit, Qiagen, Germany according to the manufacturer's protocols.

\section{HLA-B27 genotyping}

For HLA-B27 genotyping two commercial kits [HLA-B27-SSP low-resolution kit (Olerup, Sweden) and AllSet + Gold SSP B27 Low-Resolution Kit (Invitrogen, Life Technologies, Thermo Fisher Scientific, USA)] were used. All the 192 patients were successfully genotyped, while 8 controls could not be genotyped for HLA-B27, thus, the genotyping rate of the whole group of subjects (139 controls +192 patients $=331$ ) was $97.6 \%$.

\section{ERAP2 gene polymorphisms genotyping}

The two ERAP2 gene polymorphisms (rs2248374 and rs2910686) were genotyped by RT-PCR. Commercial kits TaqMan ${ }^{\circledR}$ Allelic Discrimination Assays (C_25649529_10 and C_26382310_10) were used for genotyping, according to the protocol provided by the manufacturer (Thermo Fisher Scientific Inc., Applied Biosystems TM, Foster City, MA, USA) with minor modifications (Fig. 1).

TABLE 2. The nucleotide genotyped ERAP2 polymorphisms

\begin{tabular}{|l|c|c|c|}
\hline SNPs & $\begin{array}{c}\text { Chromosome } \\
\text { position* }\end{array}$ & $\begin{array}{c}\text { Nucleotide } \\
\text { change }\end{array}$ & Gene sequence \\
\hline rs2248374 & 96900192 & A/G & Non-coding: Intron \\
\hline rs2910686 & 96916885 & C/T & Non-coding: Intron \\
\hline
\end{tabular}

*Genome Reference Consortium Human genome build 38; SNPs, single nucleotide polymorphisms.

The two polymorphisms selected for this study are located within introns, non-coding regions of the ERAP2 gene, which do not change the structure of the polypeptide molecule (Table 2). However, the impact of at least one of the polymorphisms, more specifically rs2248374 (A/G), dramatically changes the splicing process following ERAP2 transcription (43).

The splicing process is the editing process of the messenger RNA, produced immediately after transcription (pre-mRNA), which consists in the removal of introns and is needed to form the mature mRNA. Rs2248374 polymorphism is located in the vicinity of the exon 10, within the canonical splicing site 5', and directly affects pre-mRNA editing. Thus, AA genotype of rs2248374 causes the formation of a mature mRNA molecule encoding the classical ERAP2 with 960 amino acids, while the GG genotype forms a special mRNA, identified by Tanioka et al. (44), containing two TAG stop codons in the exon extension 10 and, therefore, it should encode a truncated protein of 534 amino acids. However, the in- 


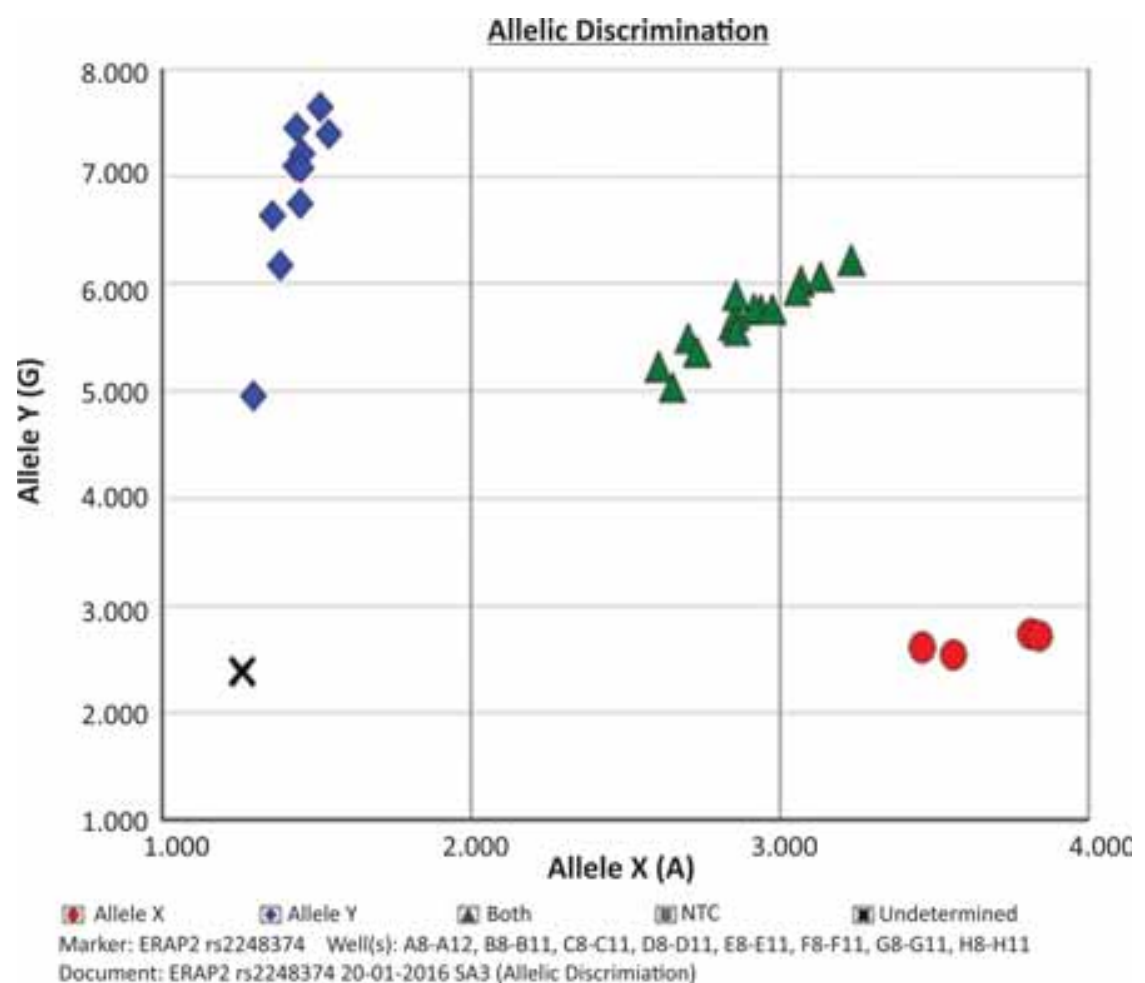

Document: ERAP2 rs2248374 20-01-2016 SA3 (Allelic Discrimiation)

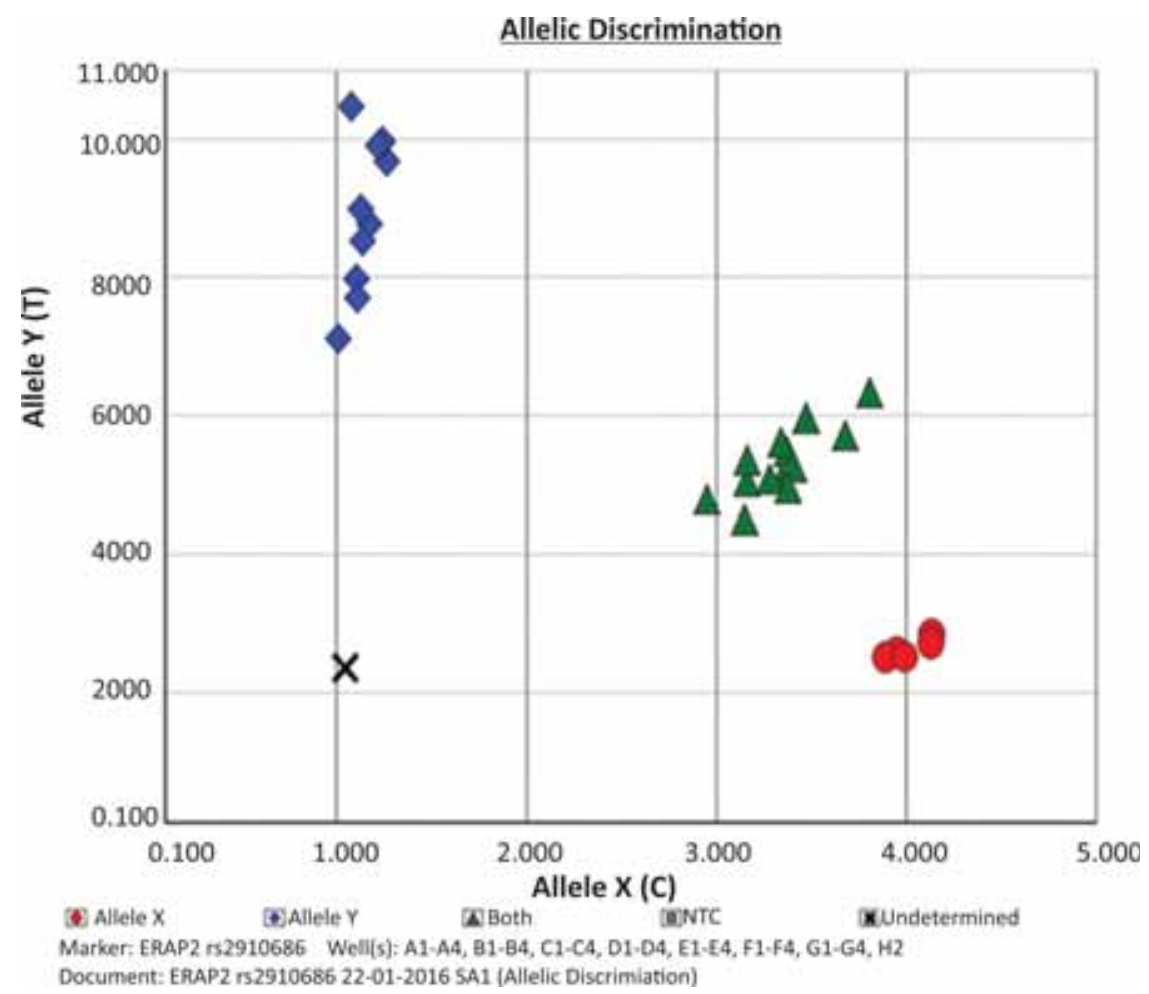

FIGURE 1. Genotyping the two polymorphisms, rs2248374 (left) and rs2910686 (right), with TaqMan ${ }^{\circledR}$ Allelic Discrimination Assays (C_25649529_10 and C_26382310_10) in AS patients. Red - AA and CC homozygotes; Green - AG and CT heterozygotes; Blue - GG and TT homozygotes; Black - NTC (no template control).

complete ERAP2 protein has not been detected. The GG genotype does not produce detectable levels of ERAP2 protein, while the AA and AG genotypes produce normal length ERAP2 (higher amount for AA genotype and lower amount for AG genotype) (43). The explanation for this apparent paradox is the degradation process of aberrant mRNA, called nonsense-mediated decay (NMD) (45). Therefore, the GG genotype carriers of rs 2248374 do not express the ERAP2 protein and, furthermore, they associate a reduced expression of MHC I molecules on B lymphocytes (43). 


\section{STATISTICAL ANALYSIS}

Hardy-Weinberg equilibrium (HWE) was assessed using Pearson's chi-squared test and the Fisher's exact test, based on the SNP-HWE algorithm proposed by the Wigginton et al. (46).

PLINK 1.9 software package (47) was used for statistical calculation of the two tests used to assess HWE. Allele and genotype frequencies were compared for each polymorphisms patients. The statistical associations were evaluated using Fisher's exact test. Statistical calculations were performed using PLINK v 1.9 (47), p values $\leq 0.05$ were considered statistically significant.

The haplotype estimation (possible alleles combinations of the two studied SNP) was performed with the software program PLINK 1.9 which uses a method based on the confidence interval implemented by Gabriel et al. (48), and the association tests between haplotype frequencies in patients and in control were performed using Pearson's chi test, using the same software PLINK 1.9 (47).

The estimation of Linkage Disequilibrium (LD) by calculating the $\mathrm{r}^{2}$ coefficient, which shows the degree of correlation between the two pairs of loci, was also performed using the program PLINK 1.9. LD reflects the correlation of neighbouring alleles derived from a single ancestral chromosome (49).

\section{RESULTS}

Both the general group of healthy controls and HLA-B27 negative controls showed no departure from Hardy-Weinberg equilibrium for both SNPs. HWE was also observed for SpA patients (general cohort and HLA-B27 negative group) for both rs2248374 and rs2910686 polymorphisms.

We compared the allelic frequencies in healthy controls of both SNPs with the ones reported by HapMap project (http://hapmap.ncbi.nlm.nih.gov) and 1000 Genomes project http://www.1000genomes.org/data) for Europeans or European descendants. Although the minor allele frequency (A) for rs2248374 was lower in our study (45.7\%) than the one reported by 1000 Genomes (48\%), no statistical significance was observed. For the second polymorphism (rs2910686) the minor allele frequency $\mathrm{C}$ in the control group was $37.4 \%$, lower than the one reported by 1000 Genome (42.6\%) and HapMap project $(48.9 \%)(\mathrm{p}<0.05)$.

There are about $17 \mathrm{~kb}$ (16693 base pairs) between the two polymorphisms (rs2248374 and rs2910686) located on chromosome 5q.15. LD estimation in healthy controls showed a moderate-high tendency of correlation between the two alleles located on adjacent loci $(\mathrm{r} 2=0.67)$.

In the first analysis we compared the alleles and genotypes frequencies between SpA patients and general controls (Table 3). Although minor allele frequency A of rs2248374 was higher in SpA patients $(51.5 \%)$ than in controls (45.7\%), this difference did not reach statistical significance $(p=0.13)$. Similarly, the five percent difference observed between minor allele $\mathrm{C}$ frequency of the second polymorphism (rs2910686) in controls (37.4\%) and SpA patients $(42.9 \%)$, did not reach the statistical threshold $(\mathrm{p}=$ $0.15)$.

Carriers of the minor allele A (AA and AG genotypes) for rs2248374 polymorphism were more fre-

TABLE 3. Minor allele and genotypes frequencies of rs2248374 and rs2910686 in SpA patients and healthy controls

\begin{tabular}{|c|c|c|c|}
\hline SNP & Controls & SpA & Statistics \\
\hline rs2248374 & $n=139$ & $n=192$ & \\
\hline \multirow{2}{*}{ Minor allele A } & Number (frequency) & Number (frequency) & \multirow{2}{*}{$\begin{array}{c}\text { OR } 1.266 \\
\text { C.I. }=[0.929-1.725] \\
p=0.13534\end{array}$} \\
\hline & 127 (45.7\%) & 198 (51.5\%) & \\
\hline Genotype AA+AG & $31+65(69 \%)$ & $49+100(77.6 \%)$ & \multirow{3}{*}{$\begin{array}{c}\text { OR } 1.552 \\
\text { C.I. }=[0.947-2.545] \\
p=0.08038\end{array}$} \\
\hline Genotype GG & $43(31 \%)$ & $43(22.4 \%)$ & \\
\hline rs2910686 & $n=139$ & $n=191$ & \\
\hline \multirow[b]{2}{*}{ Minor allele $\mathrm{C}$} & Number (frequency) & Number (frequency) & \multirow{2}{*}{$\begin{array}{c}\text { OR } 1.259 \\
\text { C.I. }=[0.917-1.727] \\
p=0.15379\end{array}$} \\
\hline & $104(37.4 \%)$ & $164(42.9 \%)$ & \\
\hline Genotype $\mathrm{CC}+\mathrm{CT}$ & $17+70(62.6 \%)$ & $37+90(66.5 \%)$ & \multirow{2}{*}{$\begin{array}{c}\text { OR } 1.186 \\
\text { C.I. }=[0.751-1.872] \\
p=0.46350\end{array}$} \\
\hline Genotype TT & $52(37.4 \%)$ & $64(33.5 \%)$ & \\
\hline
\end{tabular}

SpA, spondyloarthritides; SNP, single-nucleotide polymorphism; Cl, 95\% confidence interval; OR, odds ratio; $p$ values $\leq 0.05$ are considered significant. 
TABLE 4. The minor allele and genotypes frequencies of both studied ERAP2 polymorphisms in HLA-B27 negative SPA and healthy controls

\begin{tabular}{|c|c|c|c|}
\hline SNP & HLA-B27 negative controls & HLA-B27negative SpA & Statistics \\
\hline rs2248374 & $n=118$ & $n=101$ & \\
\hline \multirow[b]{2}{*}{ Minor allele $A$} & Number (frequency) & Number (frequency) & \multirow{2}{*}{$\begin{array}{c}\text { OR } 1.616 \\
\text { C.I. }=[1.107-2.360] \\
p=\mathbf{0 . 0 1 2 7 0}\end{array}$} \\
\hline & 105 (44.5\%) & $112(55.4 \%)$ & \\
\hline Genotype AA+AG & $25+55(67.8 \%)$ & $31+52(82.2 \%)$ & $\begin{array}{c}\text { OR } 2,190 \\
\text { C.I. }=[1.156-4.151] \\
\mathbf{p}=\mathbf{0 . 0 1 5 0 2}\end{array}$ \\
\hline Genotype GG & $38(32.2 \%)$ & $18(17.8 \%)$ & $\begin{array}{c}\text { OR } 0.382 \\
\text { C.I. }=[0.177-0.825] \\
\mathbf{p}=\mathbf{0 . 0 1 3 2 8}\end{array}$ \\
\hline rs2910686 & $\mathrm{n}=118$ & $n=100$ & \\
\hline \multirow{2}{*}{ Minor allele $\mathrm{C}$} & Number (frequency) & Number (frequency) & \multirow{2}{*}{$\begin{array}{c}\text { OR } 1.544 \\
\text { C.I. }=[1.051-2.268] \\
p=\mathbf{0 . 0 2 6 4 8}\end{array}$} \\
\hline & $85(36 \%)$ & $93(46.5 \%)$ & \\
\hline Genotype CC+CT & $13+59(61 \%)$ & $22+49(71 \%)$ & \multirow{2}{*}{$\begin{array}{c}\text { OR } 1.564 \\
\text { C.I. }=[0.886-2.762] \\
p=0.12208\end{array}$} \\
\hline Genotype TT & $46(39 \%)$ & $29(29 \%)$ & \\
\hline Genotype TT+CT & $46+59(89 \%)$ & $29+49(78 \%)$ & \multirow{2}{*}{$\begin{array}{c}\text { OR } 0.439 \\
\text { C.I. }=[0.208-0.925] \\
p=0.02773\end{array}$} \\
\hline Genotype CC & $13(11 \%)$ & $22(22 \%)$ & \\
\hline
\end{tabular}

$S N P$, single-nucleotide polymorphisms; HLA, human leukocyte antigen; SpA, spondyloarthritides; $\mathrm{Cl}, 95 \%$ confidence interval; $O R$, odds ratio; $p$ values $\leq 0.05$ are considered significant.

quent in the general group of SpA patients $(77.6 \%)$ than in controls $(69 \%)$, but the influence of disease susceptibility is uncertain. Although the p-value is higher than the statistical threshold, this value $(\mathrm{p}=$ $0.08)$ can be considered a marginal association. Larger groups of patients and controls may be needed to clarify the impact of this ERAP2 gene variation in general SpA.

When we compared the alleles and genotypes frequencies of the investigated ERAP2 polymorphisms in the HLA-B27 positive SpA group and general controls the values observed showed a similar profile in both cohorts. The minor allele A of rs2248374 was similarly expressed in patients and controls $(\mathrm{p}=0.92)$, as well as the minor allele $\mathrm{C}$ of the second SNP (rs2910686) $(\mathrm{p}=0.72)$. The genotypes of both polymorphisms had a similar distribution in both groups. Minor allele A carriers (rs2248374) and minor allele C carriers (rs2910686) did not constitute a risk factor for $\operatorname{SpA}(p=0.57$, respectively $\mathrm{p}=0.87$ ).

As we have seen, comparing the genotypes and allele frequencies of the two SNPs in general SpA group and HLA-B27 positive SpA vs. controls showed no association except for a possible marginal one for the minor allele A of rs2248374 ( $p=0.08)$. Completely different results were found when the analysis focused on HLA-B27 negative individuals alone (Table 4).

Strong associations were obtained for rs2248374. Minor allele frequency A was overexpressed (55.4\%) in HLA-B27 negative SpA group compared to HLA-B27 negative controls (44.5\%) certifying the association of this ERAP2 gene variant with $\mathrm{SpA}$ in HLA-B27 negative individuals $(\mathrm{p}=0.012)$. HLA-B27 negative carriers of the minor allele A (AA and AG genotypes) also showed an increased risk of developing seronegative spondyloarthritis $(\mathrm{p}=0.015)$. Furthermore, the GG genotype had a protective effect against the disease compared with the AA genotype $(p=0.013)$. In homozygous GG individuals for rs 2248374 polymorphism the protein levels of ERAP2 are absent due to the nonsense-mediated decay of abnormal mRNA (30).

Also for the second ERAP2 polymorphism (rs2910686), the minor allele frequency $C$ was significantly higher $(\mathrm{p}=0.02)$ in HLA-B27 negative SpA subgroup (46.5\%) compared to HLA-B27 negative controls (36\%). Although the frequency of the minor allele genotypes (CC and CT) is elevated in patients $(71 \%)$ compared with controls $(61 \%)$, it does not reach the statistical threshold $(\mathrm{p}=0.12)$. On the other hand, carriers of the major allele $\mathrm{T}$ (genotypes TT $+\mathrm{CT}$ ) are underexpressed in HLA-B27 negative SpA patients compared with HLA-B27 negative controls $(p=0.026)$, suggesting a protective effect of the wild type allele.

The analysis of reconstructed haplotypes of the two genotyped ERAP2 polymorphisms (rs2248374| rs2910686) showed somewhat expected results depending on cohorts involved. Thus, similar distribu- 
tion of the reconstructed haplotypes frequencies was observed in general groups of patients and controls (Table 5).

TABLE 5. The rs2248374|rs2910686 reconstructed haplotype frequencies in general controls and SpA cohorts

\begin{tabular}{|c|c|c|c|c|}
\hline Haplotype & Controls $(\mathbf{n}=\mathbf{1 3 9})$ & $\mathbf{S p A}(\mathbf{n}=\mathbf{1 9 2})$ & $\mathbf{C h i}^{2}$ & $\mathbf{p}$ \\
\hline AC & 0.3673 & 0.4278 & 2.435 & 0.118 \\
\hline AT & 0.0872 & 0.0892 & 0.007 & 0.930 \\
\hline GT & 0.5455 & 0.4829 & 2.498 & 0.114 \\
\hline
\end{tabular}

SpA, spondyloarthritides; $p$ values $\leq 0.05$ are considered significant.

Although minor alleles haplotype AC had a higher frequency in SpA patients $(42.78 \%)$ than in healthy controls (36.73\%), it was not a risk factor for SpA $(\mathrm{p}=0.118)$. Once we selected the HLA-B27 negative individuals in the two cohorts, the results showed a significant association of the reconstructed rs2248374|rs2910686 haplotypes with SpA.

TABLE 6. Frequencies of the reconstructed haplotypes rs2248374|rs2910686 in HLA-B27 negative patients and matching controls

\begin{tabular}{|c|c|c|c|c|}
\hline Haplotype & $\begin{array}{c}\text { HLA-B27 } \\
\text { negative controls } \\
(\mathbf{n}=\mathbf{1 1 8})\end{array}$ & $\begin{array}{c}\text { HLA-B27 } \\
\text { negative } \\
\text { SpA (n=100) }\end{array}$ & Chi $^{2}$ & $\mathbf{P}$ \\
\hline AC & 0.3519 & 0.4623 & 5.436 & $\mathbf{0 . 0 1 9}$ \\
\hline AT & 0.0901 & 0.1055 & 0.290 & 0.590 \\
\hline GT & 0.5579 & 0.4322 & 6.792 & $\mathbf{0 . 0 0 9}$ \\
\hline
\end{tabular}

SpA, spondyloarthritides; HLA, human leukocyte antigen;

$p$ values $\leq 0.05$ are considered significant.

The minor alleles AC haplotype of the two investigated SNPs was a risk factor for SpA in HLA-B27 negative individuals $(\mathrm{p}=0.019)$ (Table 6). Moreover, the major alleles GT haplotype is overexpressed in HLA-B27 negative controls (55.79\%) compared with HLA-B27 negative SpA (43.22\%), and therefore, it is a protective factor against SpA in HLA-B27 negative individuals $(\mathrm{p}=0.009)$.

\section{DISCUSSIONS AND CONCLUSIONS}

This is the first study investigating ERAP2 gene polymorphisms in a group of patients with seronegative spondyloarthritis in Romania. Moreover, although ERAP2 polymorphisms were investigated in patients with AS (27), Crohn's disease (50) or anterior acute uveitis (54) we have not find any PubMed indexed international studies analysing the ERAP2 gene's relationship with $\mathrm{SpA}$ in general. Our findings show the association of the two investigated SNPs, especially rs2248374, with SpA, but this as- sociation is restricted to HLA-B27 negative individuals. Furthermore, the reconstructed haplotypes rs2248374|rs2910686 analysis revealed minor allele $\mathrm{AC}$ haplotype as a risk factor for $\mathrm{SpA}$, while major allele GT haplotype is protective against disease in HLA-B27 negative individuals.

A variety of genetic studies have described ERAP2 gene variants as being associated with pathological conditions considered to be in close proximity to $\mathrm{SpA}$, such as: Birdshot chorioretinopathy (a rare autoimmune uveitis) (51) and psoriasis (52), or belonging to the SpA group: inflammatory bowel diseases in general (53), Crohn's disease in particular (50) and acute anterior uveitis (54). ERAP2 gene polymorphisms have been extensively studied in preeclampsia. ERAP2 contribution to this congenital pathology of the pregnancy has been confirmed in different populations: Australian and Norwegian (55), African American (56) and Chilean (57). A singular study associated ERAP2 polymorphisms with lung cancer susceptibility in Yunnan Han Chinese population (58).

Initially, the interest in ERAP2 gene in the AS susceptibility was only secondary to ERAPl gene, whose firm association with AS became evident almost ten years ago (21). Subsequently, this association was shown to be restricted to the HLA-B27 positive individuals (59). Somewhat recently, this HLA-B27 restricted association of ERAP1 with AS, but also with $\mathrm{SpA}$, has been confirmed by our group in Romanians as well (25).

In one of the first studies which investigated $E R A P 2$ in relation with AS, Tsui et al. identified in 2010 a haplotype consisting of two ERAP1 polymorphisms and one ERAP2 polymorphism (rs27044 [G] rs30187 [T] rs2549782 [T]) as being associated with a familial AS (26). The non-synonymous ERAP2 polymorphism rs2549782 is responsible for a change in the amino acid sequence (N392K) of the ERAP2 peptide and it alters both the intensity and specificity of the ERAP2 enzymatic activity (60). It is in almost complete LD with rs2248374 polymorphism, studied by us, responsible for the dramatic alteration of the splicing and the absence of the ERAP2 enzyme the endoplasmic reticulum, and therefore hardly ever translated in vivo (27).

Given the decisive effect on gene expression, in 2011 Harvey D. et al. genotyped rs 2248374 in 470 AS patients (84\% HLA-B27 positive) and 420 healthy controls and they did not report any significant differences between the two cohorts when allele 
and genotypes frequencies where compared, concluding that this ERAP2 polymorphism is not associated with AS (61). These results are similar to ours for HLA-B27 positive subgroup of SpA patients, consisting mainly of AS patients.

Recently, an International Genetics Consortium of AS (IGAS) study, involving large cohorts (more than ten thousand AS patients and more than fifteen thousand healthy controls) and using the Illumina Immunochip microarray, has identified ERAP2 gene among the non-MHC genes associated with AS (62). Three $E R A P 2$ polymorphisms were associated with AS, association which was restricted to HLA B-27 negative individuals: rs2549782 (influences the enzymatic activity of the molecule), rs 2248374 (the protective GG genotype determines undetectable ERAP2 protein levels) and rs2910686. The last two polymorphisms were genotyped in this study. Unfortunately our cohorts' size has made impossible an analysis of the SNPs in HLA-B27 negative AS patients alone. However, our findings in HLA-B27 negative SpA vs. matching controls are consistent with those reported by IGAS. Indeed, in our study the association of rs2248374 and rs2910686 polymorphisms with SpA is restricted to HLA-B27 negative individuals.

In 2015 Robinson et al. analysed subgroups of HLA-B27 positive AS-associated ERAP1 haplotypes (tagged by rs30187 and rs10050860) and either HLA-B27 positive or unselected controls of European origin from the IGAS study and they identified the association of ERAP2 polymorphism rs2248374 with AS in HLA-B27 positive individuals (27). If the association of ERAP2 with AS patients, regardless of the HLA-B27 status, is confirmed, then, the number of patients who can benefit from aminopeptidase inhibitors blocking ERAP1/ERAP2 activity, currently in research (63), will significantly increase (27).

For the understanding of AS pathogeny, the identification of ERAP1 gene's association with AS has probably been the most important milestone since the HLA-B27 discovery in the 70's and its special relation with AS. However, ERAPI continued to explain AS etiopathogeny in HLA-B27 positive patients due to the HLA-B27 positive restriction mentioned before, while too few explanations have been offered to HLA-B27 negative patients.

The discovery of ERAP2 gene among the nonMHC genes contributing to the risk of developing AS and SpA in HLA-B27 negative individuals constitutes a significant event, clarifying the etiopathogenic mechanisms for this particular subset of patients. Some ERAP2 gene variants decisively influence the enzymatic activity and therefore, they affect the antigen processing and presentation (60) on MHC class I molecules, other than HLA-B27. Recognizing the functional consequences, sometimes dramatic, of various $E R A P 2$ polymorphisms is essential for understanding disease susceptibility in HLA-B27 negative patients, but also for developing therapeutic solutions aimed to help this subgroup of patients.

\section{ACKNOWLEDGEMENTS}

Olivia Mihaela Popa was supported by the Carol Davila University of Medicine and Pharmacy Young Research Project TC 28350/04.11.2013.

\section{REFERENCES}

1. Zochling J., Brandt J., Braun J. The current concept of spondyloarthritis with special emphasis on undifferentiated spondyloarthritis. Rheumatology. 2005; 44(12):1483-91.

2. Ramanathan A., Srinivasalu H., Colbert R.A. Update on juvenile spondyloarthritis. Rheumatic diseases clinics of North America. 2013; 39(4):767-88.

3. Maxwell A., Yates M., Everden P., Gaffney K. Does the co-existence of acute anterior uveitis and chronic back pain prompt primary care physicians to consider a diagnosis of axial spondyloarthritis? Annals of the rheumatic diseases. 2016; 75(4):e18.

4. Haroon M., O'Rourke M., Ramasamy P., Murphy C.C., FitzGerald O. A novel evidence-based detection of undiagnosed spondyloarthritis in patients presenting with acute anterior uveitis: the DUET (Dublin Uveitis Evaluation Tool). Annals of the rheumatic diseases. 2015; 74(11):1990-5.

5. Zochling J., Smith E.U. Seronegative spondyloarthritis. Best Pract Res Clin Rheumatol. 2010; 24(6):747-56.

6. Braun J., Sieper J. [Spondyloarthritides]. Z Rheumatol. 2010; 69(5):425-32.

7. Stolwijk C., Boonen A., van Tubergen A., Reveille J.D. Epidemiology of spondyloarthritis. Rheumatic diseases clinics of North America. 2012; 38(3):441-76.

8. Reveille J.D. Major histocompatibility genes and ankylosing spondylitis. Best Pract Res Clin Rheumatol. 2006; 20(3):601-9.

9. Thomas G.P., Brown M.A. Genomics of ankylosing spondylitis. Discovery medicine. 2010; 10(52):263-71.

10. Braun J., Bollow M., Remlinger G., Eggens U., Rudwaleit M., Distler A., et al. Prevalence of spondylarthropathies in HLA-B27 positive and negative blood donors. Arthritis and rheumatism. 1998; 41(1):58-67.

11. Bruges-Armas J., Lima C., Peixoto M.J., Santos P., Mendonca D., da Silva B.M., et al. Prevalence of spondyloarthritis in Terceira, Azores: a population based study. Annals of the rheumatic diseases. 2002; 61(6):551-3.

12. De Angelis R., Salaffi F., Grassi W. Prevalence of spondyloarthropathies in an Italian population sample: a regional community-based study. Scandinavian journal of rheumatology. 2007; 36(1):14-21. 
13. Saraux A., Guillemin F., Guggenbuhl P., Roux C.H., Fardellone P., Le Bihan E., et al. Prevalence of spondyloarthropathies in France: 2001. Annals of the rheumatic diseases. 2005; 64(10):1431-5

14. Haglund E., Bremander A.B., Petersson I.F., Strombeck B., Bergman S., Jacobsson L.T., et al. Prevalence of spondyloarthritis and its subtypes in southern Sweden. Annals of the rheumatic diseases. 2011; 70(6):943-8.

15. Strand V., Rao S.A., Shillington A.C., Cifaldi M.A., McGuire M., Ruderman E.M. Prevalence of axial spondyloarthritis in United States rheumatology practices: Assessment of SpondyloArthritis International Society criteria versus rheumatology expert clinical diagnosis. Arthritis care \& research. 2013; 65(8):1299-306

16. Reveille J.D., Witter J.P., Weisman M.H. Prevalence of axial spondylarthritis in the United States: estimates from a crosssectional survey. Arthritis care \& research. 2012; 64(6):905-10.

17. Chaiamnuay P., Darmawan J., Muirden K.D.,

Assawatanabodee P. Epidemiology of rheumatic disease in rural Thailand: a WHO-ILAR COPCORD study. Community Oriented Programme for the Control of Rheumatic Disease. The Journal of rheumatology. 1998; 25(7):1382-7.

18. Minh Hoa T.T., Darmawan J., Chen S.L., Van Hung N., Thi Nhi C., Ngoc An T. Prevalence of the rheumatic diseases in urban Vietnam: a WHO-ILAR COPCORD study. The Journal of rheumatology. 2003; 30(10):2252-6.

19. Ng S.C., Liao Z., Yu D.T., Chan E.S., Zhao L., Gu J. Epidemiology of spondyloarthritis in the People's Republic of China: review of the literature and commentary. Seminars in arthritis and rheumatism. 2007; 37(1):39-47

20. Hukuda S., Minami M., Saito T., Mitsui H., Matsui N., Komatsubara Y., et al. Spondyloarthropathies in Japan: nationwide questionnaire survey performed by the Japan Ankylosing Spondylitis Society. The Journal of rheumatology. 2001; 28(3):554-9.

21. Burton P.R. CD, Cardon L.R., Craddock N., Deloukas P., Duncanson A., et al, for The Australo-Anglo-American Spondylitis Consortium (TASC). Association scan of 14,500 nonsynonymous SNPs in four diseases identifies autoimmunity variants. Nat Genet. 2007; 39(11):1329-37.

22. The Australo-Anglo-American Spondyloarthritis Consortium (TASC) R.J., et al. Genome-wide association study of ankylosing spondylitis identifies non-MHC susceptibility loci. Nat Genet. 2010; 42(2):123-7.

23. Evans D.M., Spencer C.C., Pointon J.J., Su Z., Harvey D., Kochan G., et al. Interaction between ERAP1 and HLA-B27 in ankylosing spondylitis implicates peptide handling in the mechanism for HLA-B27 in disease susceptibility. Nat Genet. 2011; 43(8):761-7.

24. Reveille J.D., Sims A.M., Danoy P., Evans D.M., Leo P., Pointon J.J., et al. Genome-wide association study of ankylosing spondylitis identifies non-MHC susceptibility loci. Nat Genet. 2010; 42(2):123-7.

25. Cherciu M., Popa L.O., Bojinca M., Dutescu M.I., Bojinca V., Bara C., et al. Functional variants of ERAP1 gene are associated with HLA-B27 positive spondyloarthritis. Tissue antigens. 2013; 26(10): 12158

26. Tsui F.W., Haroon N., Reveille J.D., Rahman P., Chiu B., Tsui H.W., et al. Association of an ERAP1 ERAP2 haplotype with familial ankylosing spondylitis. Annals of the rheumatic diseases. 2010; 69(4):733-6.

27. Robinson P.C., Costello M.E., Leo P., Bradbury L.A., Hollis K., Cortes A., et al. ERAP2 is associated with ankylosing spondylitis in HLA-B27-positive and HLA-B27-negative patients. 2015; 74(8):1627-9.

28. Tsujimoto M., Goto Y., Maruyama M., Hattori A. Biochemical and enzymatic properties of the M1 family of aminopeptidases involved in the regulation of blood pressure. Heart failure reviews. 2008; 13(3):285-91.

29. Tanioka T., Hattori A., Masuda S., Nomura Y., Nakayama H., Mizutani S., et al. Human Leukocyte-derived Arginine Aminopeptidase: the third member of the oxytocinase subfamily
30. Andres A.M., Dennis M.Y., Kretzschmar W.W., Cannons J.L., Lee-Lin S.Q., Hurle B., et al. Balancing selection maintains a form of ERAP2 that undergoes nonsense-mediated decay and affects antigen presentation. PLoS genetics. 2010; 6(10).

31. Hattori A., Tsujimoto M. Processing of antigenic peptides by aminopeptidases. Biological \& pharmaceutical bulletin. 2004; 27(6):777-80.

32. Evnouchidou I., Weimershaus M,. Saveanu L., van Endert P. ERAP1-ERAP2 dimerization increases peptide-trimming efficiency. J Immunol. 2014; 193(2):901-8.

33. Fruci D., Giacomini P., Nicotra M.R., Forloni M., Fraioli R., Saveanu L., et al. Altered expression of endoplasmic reticulum aminopeptidases ERAP1 and ERAP2 in transformed non-lymphoid human tissues. Journal of cellular physiology. 2008; 216(3):742-9.

34. Saveanu L., Carroll O., Lindo V., Del Val M., Lopez D., Lepelletier Y., et al. Concerted peptide trimming by human ERAP1 and ERAP2 aminopeptidase complexes in the endoplasmic reticulum. Nature immunology. 2005; 6(7):689-97.

35. Gandhi A., Lakshminarasimhan D., Sun Y., Guo H.C. Structural insights into the molecular ruler mechanism of the endoplasmic reticulum aminopeptidase ERAP1. Scientific reports. 2011; 1:186.

36. Mpakali A., Giastas P., Mathioudakis N., Mavridis I.M., Saridakis E., Stratikos E. Structural Basis for Antigenic Peptide Recognition and Processing by Endoplasmic Reticulum (ER) Aminopeptidase 2. The Journal of biological chemistry. 2015; 290(43):26021-32.

37. Rudwaleit M., Landewe R., van der Heijde D., Listing J., Brandt J., Braun J., et al. The development of Assessment of SpondyloArthritis international Society classification criteria for axial spondyloarthritis (part I): classification of paper patients by expert opinion including uncertainty appraisal. Annals of the rheumatic diseases. 2009; 68(6):770-6.

38. Rudwaleit M., van der Heijde D., Landewe R., Listing J., Akkoc N., Brandt J., et al. The development of Assessment of SpondyloArthritis international Society classification criteria for axial spondyloarthritis (part II): validation and final selection. Annals of the rheumatic diseases. 2009; 68(6):777-83.

39. Rudwaleit M., van der Heijde D., Landewe R., Akkoc N., Brandt J., Chou C.T., et al. The Assessment of SpondyloArthritis International Society classification criteria for peripheral spondyloarthritis and for spondyloarthritis in general. Annals of the rheumatic diseases. 2011; 70(1):25-31.

40. Dougados M., van der Linden S., Juhlin R., Huitfeldt B., Amor B., Calin A., et al. The European Spondylarthropathy Study Group preliminary criteria for the classification of spondylarthropathy. Arthritis and rheumatism. 1991; 34(10):1218-27.

41. van der Linden S., Valkenburg H.A., Cats A. Evaluation of diagnostic criteria for ankylosing spondylitis. A proposal for modification of the New York criteria. Arthritis and rheumatism. 1984; $27(4): 361-8$.

42. Taylor W., Gladman D., Helliwell P., Marchesoni A., Mease P., Mielants H. Classification criteria for psoriatic arthritis: development of new criteria from a large international study. Arthritis and rheumatism. 2006; 54(8):2665-73.

43. Andrés A.M., Dennis M.Y., Kretzschmar W.W., Cannons J.L., Lee-Lin S-Q., Hurle B., et al. Balancing Selection Maintains a Form of ERAP2 that Undergoes Nonsense-Mediated Decay and Affects Antigen Presentation. PLoS genetics. 2010; 6(10):e1001157.

44. Tanioka T., Hattori A., Masuda S., Nomura Y., Nakayama H., Mizutani S., et al. Human leukocyte-derived arginine aminopeptidase. The third member of the oxytocinase subfamily of aminopeptidases. The Journal of biological chemistry. 2003; 278(34):32275-83

45. Pinyol M., Bea S., Pla L., Ribrag V., Bosq J., Rosenwald A., et al. Inactivation of RB1 in mantle-cell lymphoma detected by nonsense-mediated mRNA decay pathway inhibition and microarray analysis. Blood. 2007; 109(12):5422-9.

46. Wigginton J.E., Cutler D.J., Abecasis G.R. A note on exact tests of Hardy-Weinberg equilibrium. American journal of human genetics. 2005; 76(5):887-93. 
47. Chang C.C., Chow C.C., Tellier L.C., Vattikuti S., Purcell S.M., Lee J.J. Second-generation PLINK: rising to the challenge of larger and richer datasets. GigaScience. 2015;4:7.

48. Gabriel S.B., Schaffner S.F., Nguyen H., Moore J.M., Roy J., Blumenstiel B., et al. The structure of haplotype blocks in the human genome. Science (New York, NY). 2002; 296(5576):2225-9.

49. Reich D.E., Cargill M., Bolk S., Ireland J., Sabeti P.C., Richter D.J., et al. Linkage disequilibrium in the human genome. Nature. 2001; 411(6834):199-204.

50. Franke A., McGovern D.P., Barrett J.C., Wang K., RadfordSmith G.L., Ahmad T., et al. Genome-wide meta-analysis increases to 71 the number of confirmed Crohn's disease susceptibility loci. Nat Genet. 2010; 42(12):1118-25.

51. Kuiper J.J., Van Setten J., Ripke S., Van T.S.R., Mulder F., Missotten T., et al. A genome-wide association study identifies a functional ERAP2 haplotype associated with birdshot chorioretinopathy. Human molecular genetics. 2014; 23(22):6081-7.

52. Tsoi L.C., Spain S.L., Knight J., Ellinghaus E., Stuart P.E., Capon F., et al. Identification of 15 new psoriasis susceptibility loci highlights the role of innate immunity. Nat Genet. 2012; 44(12):1341-8.

53. Jostins L., Ripke S., Weersma R.K., Duerr R.H., McGovern D.P., Hui K.Y., et al. Host-microbe interactions have shaped the genetic architecture of inflammatory bowel disease. Nature. 2012 491(7422): 119-24

54. Robinson P.C., Claushuis T.A., Cortes A., Martin T.M., Evans D.M., Leo P., et al. Genetic dissection of acute anterior uveitis reveals similarities and differences in associations observed with ankylosing spondylitis. Arthritis \& rheumatology (Hoboken, $\mathrm{NJ}$ ). 2015; 67(1):140-51.

55. Johnson M.P., Roten L.T., Dyer T.D., East C.E., Forsmo S., Blangero J., et al. The ERAP2 gene is associated with preeclampsia in Australian and Norwegian populations. Human genetics. 2009; 126(5):655-66.

56. Hill L.D., Hilliard D.D., York T.P., Srinivas S., Kusanovic J.P., Gomez R., et al. Fetal ERAP2 variation is associated with preeclampsia in African Americans in a case-control study. BMC medical genetics. 2011;12:64.
57. Vanhille D.L., Hill L.D., Hilliard D.D., Lee E.D., Teves M.E., Srinivas S., et al. A Novel Haplotype Structure in a Chilean Population: Implications for ERAP2 Protein Expression and Preeclampsia Risk. Molecular genetics \& genomic medicine. 2013; 1(2):98-107.

58. Zhou J., Ma Q., Guo C., Shi L., Yao Y., Yu J., et al. [A study on the relationship between endoplasmic reticulum aminopeptidase2 genetic polymorphisms and non-small cell lung cancer in Yunnan Han population]. Zhonghua jie he he hu xi za zhi = Zhonghua jiehe he huxi zazhi $=$ Chinese journal of tuberculosis and respiratory diseases. 2014; 37(12):909-14.

59. The Australo-Anglo-American Spondyloarthritis Consortium (TASC) ED, et al. Interaction between ERAP1 and HLA-B27 in ankylosing spondylitis implicates peptide handling in the mechanism for HLA-B27 in disease susceptibility. Nat Genet. 2011; 43(8):761-7.

60. Evnouchidou I., Birtley J., Seregin S., Papakyriakou A., Zervoudi E., Samiotaki M., et al. A common single nucleotide polymorphism in endoplasmic reticulum aminopeptidase 2 induces a specificity switch that leads to altered antigen processing J Immunol. 2012; 189(5):2383-92.

61. Harvey D., Pointon J.J., Karaderi T., Appleton L.H., Farrar C., Wordsworth B.P. A common functional variant of endoplasmic reticulum aminopeptidase 2 (ERAP2) that reduces major histocompatibility complex class I expression is not associated with ankylosing spondylitis. Rheumatology (Oxford, England). 2011; 50(9):1720-1.

62. Cortes A., Hadler J., Pointon J.P., Robinson P.C., Karaderi T., Leo P., et al. Identification of multiple risk variants for ankylosing spondylitis through high-density genotyping of immune-related loci. Nat Genet. 2013; 45(7):730-8.

63. Zervoudi E., Saridakis E., Birtley J.R., Seregin S.S., Reeves E., Kokkala P., et al. Rationally designed inhibitor targeting antigentrimming aminopeptidases enhances antigen presentation and cytotoxic T-cell responses. Proceedings of the National Academy of Sciences of the United States of America. 2013;110(49):19890-5. 